\title{
Personalização de Conteúdo e Avaliação Multicritério em Ambiente Virtual de Aprendizagem de Código Aberto
}

\author{
Douglas Rosemann ${ }^{1}$, André L. A. Raabe ${ }^{1}$, Raimundo C. Ghizoni Teive ${ }^{1}$ \\ ${ }^{1}$ Curso de Mestrado Acadêmico Em Computação Aplicada \\ Universidade do Vale do Itajaí (UNIVALI) - Itajaí, SC - Brasil \\ d.rosemann@gmail.com, raabedunivali.br, rteive@univali.br
}

\begin{abstract}
The Virtual Learning Environments (VLE) are develop to support the teaching-learning process. The student interacts with the environment, and it supervises the students, guiding their studies, through the interaction of the teacher as a tutor or through intelligent tools. The VLE Moodle stands out for being open and adaptable code, which allows exploring the application of an Intelligent Tutoring System (ITS) with insertion of a technique of Artificial Intelligence (AI) called Bayesian Networks (BN), enabling customized instruction. This AVA has the functionality to record some information from student interaction with the environment. Some of this information will be classified as criteria and applied within the concept of the Multi Criteria Evaluation and thereby measure the level of student learning.
\end{abstract}

Resumo. Os Ambientes Virtuais de Aprendizagem (AVA) são desenvolvidos para dar suporte ao processo de ensino-aprendizagem. O aluno interage com o ambiente, e o mesmo orienta os estudantes na condução dos seus estudos, através da interação do professor como tutor ou através de ferramentas inteligentes. O AVA Moodle se destaca por ser de código aberto e adaptável, o que permite explorar a aplicação de um Sistema Tutor Inteligente (STI) com inserção de uma técnica de Inteligência Artificial (IA) denominada Redes Bayesianas (RB), possibilitando assim instrução customizada. Este AVA possui a funcionalidade de registrar algumas informações da interação do aluno com o ambiente. Algumas destas informações serão classificadas como critérios e aplicadas dentro do conceito da Avaliação Multicritério e assim mensurar o nível de aprendizado de um estudante.

\section{Introdução}

Os Ambientes Virtuais de Aprendizagem (AVA) são desenvolvidos para dar suporte a processos de aprendizagem, tanto nas modalidades de ensino presencial como a distância. Um AVA agrupa em um espaço virtual, recursos definidos em meios eletrônicos, como: fórum, wiki, bate-papo, conferências, envios de mensagens, material de leitura, banco de questões, e outras tecnologias que colaboram para o processo de ensino e aprendizagem. Este ambiente é configurado com a meta de alcançar determinados objetivos definidos pelo tutor do ambiente.

Com uma estratégia adequada, um AVA pode fomentar a autonomia dos estudantes, pelo fato do mesmo poder acessar o ambiente em horários variados, e o uso de um tutor inteligente pode oportunizar outras estratégias pedagógicas, podendo propiciar novas oportunidades de ensino. 
O desenvolvimento de um AVA exige a criação de uma plataforma na qual o aluno interaja, e que a partir desta interação o ambiente reaja. Esta reatividade pode ser realizada por um professor tutor ou por ferramentas inteligentes. E nesta interação, é que se espera que ocorra uma colaboração positiva no processo de ensino e aprendizagem.

Já existem vários AVAs consolidados no mercado, entre eles é possível identificar alguns com maior aplicabilidade perante outras soluções. Um AVA que se destaca devido a sua particularidade de ser de código-aberto e possuir bibliotecas adicionais para adaptação é o Moodle. Como destacam Al-Ajlan e Zedans (2008), o Moodle tem uma comunidade para troca de ideias, possibilidades para adaptação, além da aplicação em universidades.

O Moodle fornece suporte para disponibilização de conteúdos, porém não está preparado para aplicar adaptação destes conteúdos conforme o nível de conhecimentos daqueles que o utilizam. Este AVA também é responsável em registrar informações da interação do aluno com o ambiente, porém a extração destas informações exige o conhecimento da estrutura de banco de dados e de que forma estas informações são armazenadas, para assim conseguir recuperar informações úteis sobre a interação do estudante.

Assim seria interessante substituir o modelo tradicional de EAD, caracterizado pelo predomínio do conteúdo informativo, por outro paradigma, centrado na ação educativa flexível, aberta e interativa, para que o aluno percorra seu ritmo individual, de forma autônoma, pois como colocado em Netto, Giraffa e Faria (2010), os alunos aprendem de formas diferenciadas, e assim é necessário diversificar as estratégias e os materiais utilizados.

Através da Revisão Sistemática da Literatura (RSL) foi possível observar que alguns trabalhos pesquisados propõem o uso de técnicas da IA para condução dos conteúdos, no sentido da indicação do sequenciamento destes conteúdos. Porém, não adaptam AVA tradicionais, nem consideram uma avaliação sob o ponto de vista de múltiplos critérios, buscando ter uma avaliação mais global do aluno e não somente baseada em testes. Exemplos destes trabalhos são: Nunes, Mueller e Teive (2008), Suebnukam e Haddawy (2007), Acampona; Gaeta e Loia (2010), Aguillar et al. (2011), Crysafiadi e Virvou (2012), Noh et al. (2012), Badaracco e Martínez (2013).

Outros autores como Wilges et al. (2012), referenciam a utilização de plataforma já existente, adaptando-os para sugestão de conteúdo, mas sem mencionar os detalhes de como os estudantes são avaliados.

Neste sentido, é proposto neste trabalho um Sistema Tutor Inteligente para ser utilizado com o Moodle, no qual a personalização do conteúdo é feita através de uma Rede Bayesiana e a avaliação de desempenho dos alunos é executado por um método multicritério.

A personalização de conteúdo em um AVA pode auxiliar o estudante na condução de seus estudos, atuando como um guia ou tutor, focando naquilo que é relevante ele saber naquele momento, buscando obter resultado satisfatório no seu teste de conteúdo atual e consequentemente no avanço do assunto a ser dominado. Neste sentido, é aplicado o STI como guia do conteúdo a ser estudado pelo aluno. 


\section{Projeto do STI Proposto}

A personalização de conteúdo em um AVA pode auxiliar o estudante na condução de seus estudos, atuando como um guia ou tutor, focando naquilo que é relevante ele saber naquele momento, buscando obter resultado satisfatório no seu teste de conteúdo atual e consequentemente no avanço do assunto a ser dominado. Neste sentido, é aplicado o STI como condutor de conteúdo.

Para a aplicação da AM, serão consideradas as informações registradas pelo STI, como também as informações acumuladas no AVA, ao que se refere a interação do estudante com o ambiente. Assim com base nos critérios, foi implementada uma função de agregação aditiva que será apresentado ao tutor do ambiente, na forma de auxílio complementar para definir o nível de aprendizado deste estudante. Para mensurar o resultado da AM, a mesma será comparada com as notas dos testes de conteúdo e informações registradas no AVA dos alunos voluntários, e assim analisar através de notas calculadas, os resultados gerados pela interação destes alunos com o ambiente.

O desenvolvimento parte do AVA Moodle, e dentro deste ambiente é estruturado um STI que fará a análise dos testes de conteúdo. Quando o aluno solicitar tutoria, o STI buscará o resultado dos testes, executará a inferência na $\mathrm{RB}$, que na sequencia retornará a decisão quanto a nova apresentação de conteúdo dentro do AVA. Para o professor tutor, o AVA disponibiliza acesso a avaliação multicritério, separado por aluno e critério avaliado.

\subsection{Desenvolvimento no AVA}

O desenvolvimento no AVA será através da geração de um bloco, que é uma das possibilidades de código aberto que o Moodle permite gerenciar, e sobre este bloco o estudante fará a solicitação para a geração da inferência da RB. O professor utilizará o mesmo bloco que trará informações gerenciais dos estudantes referente a disciplina ministrada. A Figura 1 representa a relação das técnicas a serem utilizadas na implementação do STI.

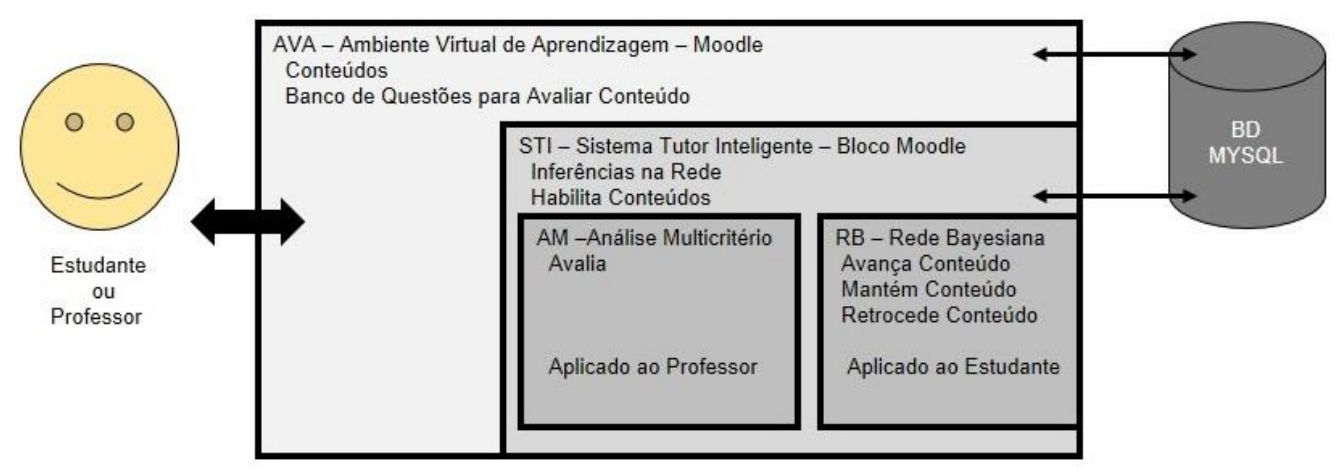

Figura 1. A Organização da Estrutura do AVA com STI

Com relação à Figura 1, tem-se:

- Estudante ou Professor: responsáveis pela interação com o AVA. O professor fica responsável em disponibilizar o curso em forma de tópicos, e cada tópico conterá o conteúdo a ser estudado. Além disso o professor disponibiliza os testes de 
conteúdo classificados por nível de dificuldade: fácil, médio e difícil, sendo que o resultado da AM ficará disponível ao professor para acompanhamento.

- AVA - Ambiente Virtual de Aprendizagem - Moodle: É o AVA especificamente apresentado, responsável em armazenar e apresentar os conteúdos e avaliações de um modo geral;

- Banco de Dados (BD) - MYSQL: Responsável em armazenar as informações dos alunos e professores, seus dados e interações com o ambiente;

- STI - Sistema Tutor Inteligente - Bloco Moodle: Denominado Bayes Criteria, e será responsável em habilitar os conteúdos conforme o estudante interage com o AVA. Analisará o resultado da avaliação do estudante, gerando inferência na rede com o resultado das avaliações e adaptando o conteúdo da disciplina em questão;

- AM - Avaliação Multicritério: sumariza as informações contidas no AVA e no STI através do Banco de Dados (BD) e gera a Avaliação Multicritério;

- RB - Rede Bayesiana: voltado especificamente para o aluno. É responsável em receber as informações referente a avaliação de conteúdo e então sugerir para que seja avançado, mantido ou retrocedido o conteúdo.

\subsection{Rede Bayesiana Implementada}

As Redes Bayesianas (RB), são técnicas da Inteligência Artificial para a modelagem probabilística do conhecimento. A modelagem de uma RB pode ser aplicada ao tratamento de informações que não se possui certeza sobre a resposta. Neste sentido, o perfil do estudante está associado ao conhecimento do mesmo, quais são suas preferências, dificuldades e em qual ponto é possível melhorar o desempenho, sendo por isso foco do STI proposto neste trabalho.

As associações das técnicas descritas até o momento, serão empregadas sobre a disciplina de Estrutura de Dados (ED), que dentro da área da computação, é responsável pelo estudo dos diversos mecanismos de organização de dados, para atender aos diferentes requisitos de processamento. Os tópicos da disciplina de ED selecionados são: Listas, Pilhas, Filas, Árvores, Métodos de Pesquisa e Métodos de Ordenação. Para reforço da disciplina, foram selecionados os tópicos: Vetores, Alocação Dinâmica e Recursividade. Estes tópicos fazem parte da disciplina de Algoritmos e Programação de Computadores, disciplina que é pré-requisito de ED. Todos estes tópicos serão ministrados pela RB conforme o avanço ou retrocesso no estudo do aluno pelo ambiente.

A modelagem do conteúdo da disciplina de ED, estará constituído na forma de nós da RB. A inferência sobre a rede de nós, ocorrerá através da aplicação do resultado dos testes sobre o conteúdo estudado. A RB utilizada neste estudo, deriva do trabalho elaborado por Nunes, Mueller e Teive (2008) que sugere o uso de uma RB para recomendação de conteúdo, sendo que deste trabalho obteve-se as Tabelas de Probabilidades Condicionais (TPC).

Esta tabela contém para cada nó, valores numéricos com a probabilidade $a$ posteriori para cada caso condicional proveniente dos nós pais. Todas as entradas da matriz de probabilidades conjunta podem ser calculadas a partir de informações disponíveis na RB. Então, cada entrada da matriz é representada pelo produto dos elementos adequados das TPC. Em Nunes, Mueller e Teive (2008), para simular os testes necessários, optou-se pelo desenvolvimento de um AVA específico para disponibilização de conteúdo e seus respectivos testes. Enquanto que neste trabalho, optou-se em aplicar 
esta TPC sobre um AVA open source já disponível, e que juntamente aos testes, abordará a aplicação de outros critérios para mensurar o nível de aprendizado do estudante.

A Figura 2 representa a RB da disciplina de Estrutura de Dados. Sua elaboração foi possível através da utilização de uma API denominada Genie 2.0.

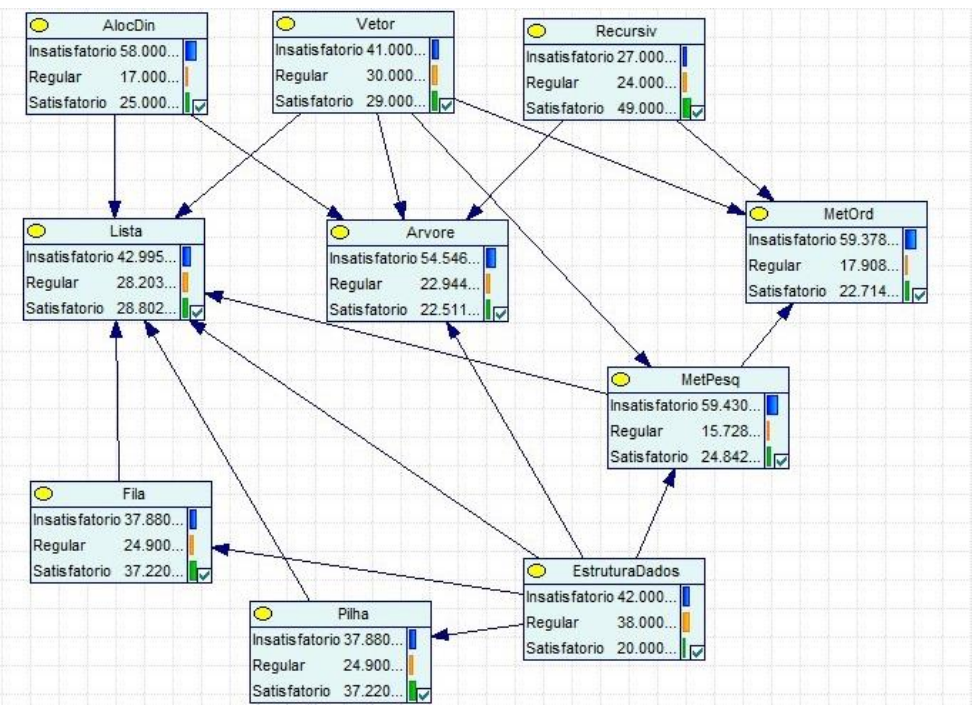

Figura 2. RB da disciplina de Estrutura de Dados

Após o aluno finalizar a avaliação de conteúdo, é apresentada uma nota representando os acertos. A classificação da nota é representada pela Tabela 1, e o seu resultado é a evidência a ser aplicada na RB:

Tabela 1. Classificação das Evidências

\begin{tabular}{|l|l|}
\hline Classificação & Faixa de Acerto \\
\hline Insatisfatório & Para acertos $<60 \%$ \\
\hline Regular & Para $60 \% \leq$ acertos $\leq 70 \%$ \\
\hline Satisfatório & Para acertos $>70 \%$ \\
\hline
\end{tabular}

Com a classificação definida, a mesma é indicada na rede como evidência para identificar as probabilidades dos nós conforme o valor da classificação. No caso de regular e insatisfatório, a probabilidade pode ser consultada para ativar algum reforço. No caso de satisfatório a rede pode ser consultada para identificar em qual dos próximos tópicos da disciplina o aluno pode ter maior dificuldade e assim formalizar alguma ação.

\subsection{Avaliação Multicritério}

Outro aspecto relevante que pode ser incorporado a um AVA ou STI, é a avaliação de desempenho do estudante no domínio dos conteúdos disponibilizados e no uso destes ambientes como um todo. Neste caso, existem diversos critérios que podem ser considerados para avaliação deste desempenho. É possível citar como possíveis critérios de avaliação: os acessos do aluno no ambiente, os acessos à disciplina e o tempo de execução de avaliação. Entretanto, de forma geral, esta avaliação pode tornar-se muito subjetiva, sendo a sua modelagem um desafio adicional.

Desta forma, a aplicação da avaliação multicritério (AM) permite o emprego de critérios e definição de sua importância através de pesos. Neste estudo, por meio da interação do estudante no AVA, pretende-se aplicar alguns critérios para avaliar o 
desempenho do aluno de uma forma mais global. Neste estudo, foram formalizados quatro critérios que serão mensurados através da avaliação multicritério e indicarão um valor numérico na escala de zero a dez, o nível de aprendizado do estudante.

Tabela 2. Classificação das Evidências

\begin{tabular}{|l|l|}
\hline Critério & Significado Conteúdo/Avaliação \\
\hline $\begin{array}{l}\text { Nota dos Testes de } \\
\text { Conteúdo }\end{array}$ & $\begin{array}{l}\text { A nota do teste serve como referência de entendimento do } \\
\text { conteúdo no momento que é aplicada a avaliação. Será utilizada } \\
\text { a nota do teste para geração do nível de aprendizado. }\end{array}$ \\
\hline $\begin{array}{l}\text { Número Acessos } \\
\text { Ambiente }\end{array}$ & $\begin{array}{l}\text { A quantidade de acessos ao ambiente caracteriza que o estudante } \\
\text { está procurando o ambiente para estudos. }\end{array}$ \\
\hline $\begin{array}{l}\text { Número Acessos } \\
\text { Disciplina }\end{array}$ & $\begin{array}{l}\text { A quantidade de vezes que acessou a disciplina para verificar } \\
\text { buscou o material para estudo antes de efetuar a avaliação. }\end{array}$ \\
\hline $\begin{array}{l}\text { Tempo Execução } \\
\text { do teste de } \\
\text { Conteúdo }\end{array}$ & $\begin{array}{l}\text { O tempo que o estudante leva para fazer a avaliação, demonstra } \\
\text { o nível de raciocínio e formação de resposta. }\end{array}$ \\
\hline
\end{tabular}

Para alcançar o nível de aprendizado do estudante através da AM, é necessário executar algumas etapas importantes para o processo decisório, através da Figura 3, as etapas são visualizadas e em seguida descritas:

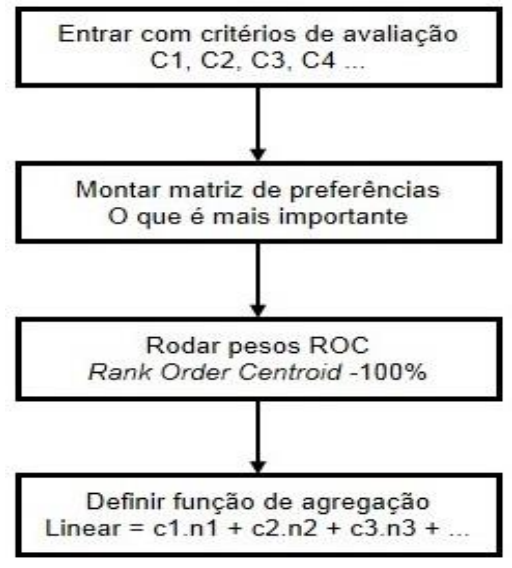

Figura 3. Estruturação da AM

Cada etapa da Figura 3 é especificada abaixo:

- Entrada com os critérios de avaliação: esta entrada é escolhida pelo professor no momento de configurar a estrutura da disciplina no AVA. A priori serão disponibilizados os critérios especificados pela Tabela 2;

- Montar matriz de preferências: O tutor na etapa de definição de critérios deverá selecionar a importância de critérios, no sentido de definir o de maior importância para o menor;

- Rodar pesos Rank Order Centroid (ROC): Como não é sabido o valor dos pesos exatos, estes serão definidos pela metodologia ROC que utilizam como base o cálculo do centroide, propostos por Barron (1992), a metodologia ROC são derivados de uma análise sistemática da informação implícita no ranque da preferência ordinal dos critérios. Os pesos são determinados pelas médias das coordenadas correspondentes aos vértices que definem o centroide. Os pesos ROC são calculados a partir dos vértices do simplex $\mathcal{S}_{n}$, definido por $w_{1} \geq w_{2} \geq \cdots \geq$ 
$w_{n} \geq 0$ e restrito por $\sum_{i=1}^{n} w_{i}=1$, cujos vértices são $\boldsymbol{e}_{\mathbf{1}}=(1,0, \cdots, 0), \boldsymbol{e}_{\mathbf{2}}=$ $(1 / 2,1 / 2,0, \cdots, 0), \quad \boldsymbol{e}_{3}=(1 / 3,1 / 3,1 / 3,0, \cdots, 0), \quad \ldots, \quad \boldsymbol{e}_{n}=$ $(1 / n, 1 / n, 1 / n, \cdots, 1 / n)$. De forma geral, para o i-ésimo atributo mais importante, o peso centroide é calculado pela equação:

$$
w_{i}(R O C)=\frac{1}{n} \sum_{j=i}^{n} \frac{1}{j}, \quad i=1, \cdots, n .
$$

- Definir função de agregação: Esta função de agregação terá por objetivo definir uma nota através de uma função linear, aplicando os pesos e as notas alcançadas de cada critério selecionado.

\subsection{Implementação}

Como o Moodle é implementado na linguagem PHP, o Bloco que contém o STI foi desenvolvido na mesma linguagem. Este bloco foi desenvolvido tendo duas separações em seu código, uma parte para atender as requisições do estudante no que se refere a tutoria, e em outra parte do código para atender as requisições do tutor quanto à avaliação multicritério. Porém as inferências sobre a RB tiveram que ser desenvolvidas na linguagem Java, pois a biblioteca aplicada para esta operação denominada JSmile, foi desenvolvida nesta linguagem de programação. Para conseguir integrar as aplicações destas duas linguagens, foi aplicado o conceito de webservices.

Assim, com a criação do webservice, o mesmo recebe como parâmetro o nome do nó da RB e sua evidência, e conforme estes valores passados, o resultado será uma lista de nós a serem estudados ou reforçados conforme a RB interpretar o resultado da evidência. A comunicação do webservice é intermediada pelo Apache Tomcat 7.0 que conseguiu estabelecer a comunicação efetiva entre os projetos das duas linguagens.

\section{Resultados Alcançados}

Os primeiros resultados apontam para a aplicação efetiva da RB dentro do AVA, como também a geração das primeiras informações da AM. Especificamente a RB proporciona a geração de um ambiente com possibilidade de adaptação de conteúdo, respeitando o resultado dos testes de conteúdo realizados pelo aluno. $\mathrm{O}$ ensaio foi aplicado com seis voluntários que foram conduzidos pelo experimento duplo cego. Para isto foram definidos dois grupos com três pessoas cada, no qual um grupo fez os testes conduzido pelo STI e o outro grupo foi conduzido pelo AVA, porém sem interferência do STI. Em todas estas situações o AVA registra o resultado da interação, mas a RB só é invocada pelo grupo que possui o STI ativado. O ambiente selecionado é o Moodle na versão 2.6.1, e foi aplicado um dos conceitos de adaptação disponíveis neste AVA que é a criação de blocos. Assim sendo, foi criado um bloco, que para este projeto ficou denominado como Bayes Criteria.

Logo que o aluno entra no ambiente, o mesmo é convidado a solicitar tutoria para iniciar o processo de estudos. Para o voluntário sem a condução do STI, todos os tópicos e avaliações são disponibilizados, sendo o aluno responsável pelo avanço ou retrocesso. Para os voluntários conduzidos pelo STI, é apresentado o somente o tópico listas para estudos. Continuando o processo, o próximo passo será a execução dos testes de 
conteúdo. Ao finaliza-lo é solicitado pelo estudante a tutoria do STI que analisará o resultado deste teste conforme a Tabela 1 . Na situação insatisfatória ou regular, poderá ser ativado um dos tópicos de reforços conforme a inferência na RB. Esta inferência gera como retorno o reforço selecionado e solicitado através de mensagem descritiva no bloco para que faça novamente a avaliação do mesmo nível. Quando na condição satisfatória é solicitado que faça na sequência, os testes de conteúdo médio e difícil. Ao final de cada teste deve-se solicitar a tutoria. A troca de tópico está condicionada na execução dos testes dos três níveis com aproveitamento satisfatório. A Figura 5 representa o estágio inicial de estudo.
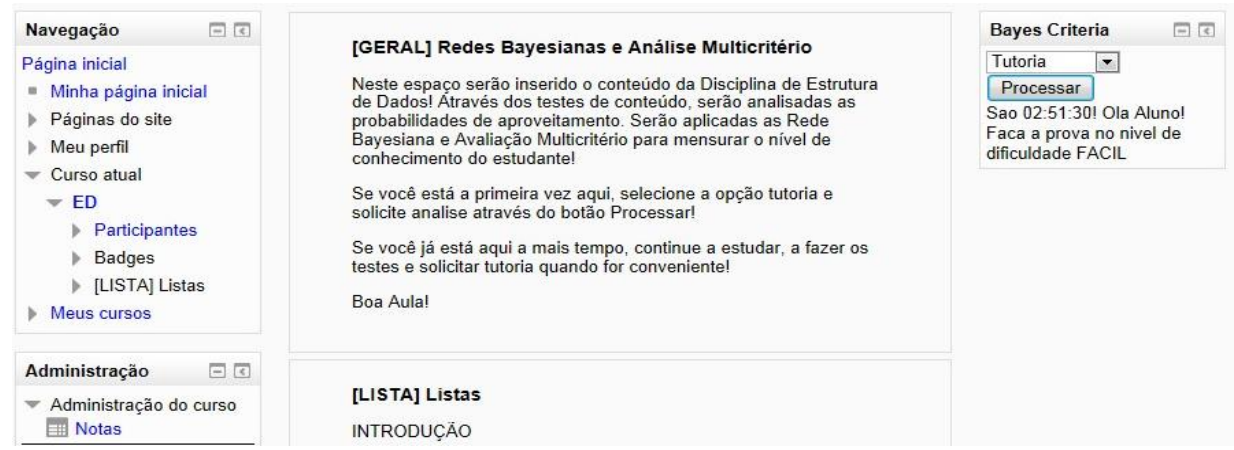

Figura 4. AVA Moodle com Bloco Bayes Criteria e tópico LISTA

Nessa simulação o resultado da avaliação foi considerado $50 \%$ de acertos, logo, ao solicitar tutoria, o STI solicita a RB uma análise do nó Listas com probabilidade de $50 \%$ que equivale a insatisfatório. O resultado da rede sugere o estudo do nó de reforço Alocação Dinâmica, pois somando a probabilidade Insatisfatório e Regular, resulta como sendo a maior probabilidade de dificuldade a posteriori do que o somatório das mesmas probabilidades do nó vetor. Esta conclusão está representada pela própria RB na Figura 6. Enquanto que a visualização do reforço habilitado no AVA é representada na Figura 7. Para esta etapa, a RB proporciona a efetiva possibilidade de adaptação de conteúdo.

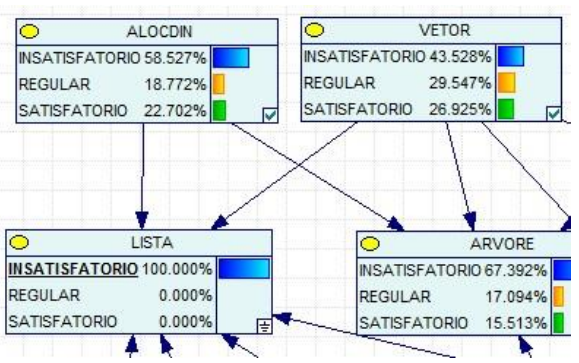

Figura 5. RB com Evidência em LISTA e probabilidades de ALOCDIN e VETOR
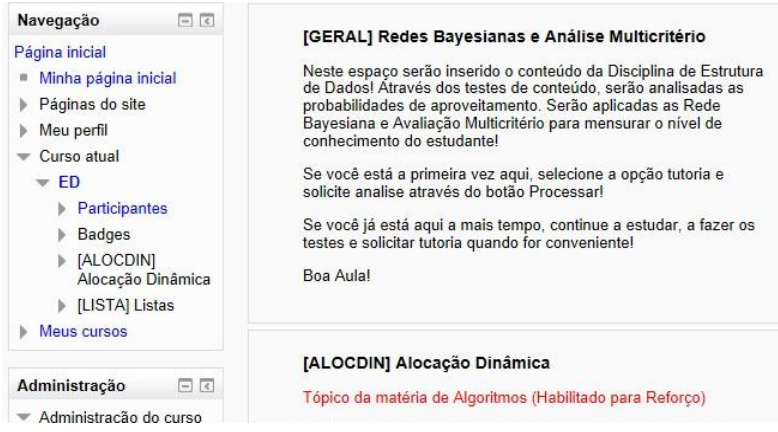


\section{Figura 6. AVA Moodle com Bloco Bayes Criteria e tópico de Reforço ALOCDIN}

A AM gerou os primeiros resultados quanto a extração de informações da interação dos alunos com o ambiente. Foi possível também validar a metodologia ROC responsável pela geração dos pesos. As notas dos critérios foram implementas analisando o resultado da consulta no banco de dados de cada critério, e conforme o resultado desta consulta, foi então aplicada uma regra de três com limite mínimo e máximo para gerar a nota. Esta regra foi codificada diretamente no projeto, sendo possível adaptação somente por código implementado. Conforme a Figura 8 é possível observar os critérios classificados em ordem de preferência, como também os pesos gerados pela metodologia ROC. Na sequência as notas calculadas de cada critério e a nota parcial. Ao final é apresentada uma média geral, resultado da soma das notas parciais.

\begin{tabular}{|c|c|c|c|}
\hline Criterio & Peso & Nota Criterio & Nota Parcial \\
\hline Nota dos Testes & 0.52083333333333 & 10 & 5.2083333333333 \\
\hline Tempo de Avaliação Questões & 0.27083333333333 & 0 & 0 \\
\hline Tempo no Ambiente & 0.14583333333333 & 10 & 1.4583333333333 \\
\hline Quantidade de Acesso a Disciplina & 0.0625 & 10 & 0.625 \\
\hline
\end{tabular}

Média Geral pela Avaliação Multi-Critério 7.2916666666667

Figura 7. Avaliação multicritério gerada através da interação de um aluno

Ainda será solicitada a análise de um professor para aferir a avaliação multicritério quanto a geração dos resultados fornecidos pelo bloco. Os alunos avaliados também responderam a um questionário de satisfação, para assim avaliar o nível de aceitação proposto pela adaptação de conteúdo. Este questionário será considerado como informação adicional para as considerações finais deste trabalho.

\section{Considerações Finais}

O desenvolvimento de um AVA requer a criação de uma plataforma na qual o aluno possa interagir, e que a partir desta interação o ambiente possa fazer o papel de um professor, através de um STI, o qual usualmente é baseado em técnicas de inteligência artificial. Para este trabalho foi selecionado o AVA Moodle, sendo implementado um STI, via criação de blocos. Assim, com a funcionalidade de blocos foi desenvolvido um Sistema Tutor Inteligente, associado a uma Rede Bayesiana (RB) para a disciplina de Estrutura de Dados do curso de Ciência da Computação, sendo esta RB responsável por gerar a adaptação de conteúdo.

Além disto, com o objetivo de mensurar o nível de aprendizado do estudante, foi desenvolvido um bloco no Moodle para Avaliação Multicritério. Os critérios já foram pré-selecionados, sendo: nota dos testes de conteúdo, acesso ao ambiente, acesso a disciplina e tempo de execução dos testes de conteúdo. Estes critérios são ordenados através de uma matriz de preferências, e o peso de cada critério será calculado através da aplicação da Metodologia Rank Order Centroid. Esta metodologia gera um valor para formação de uma nota de zero a dez, e assim representar o nível de aprendizado do estudante.

Os primeiros resultados obtidos indicam a viabilidade de combinação das técnicas. As simulações realizadas possibilitaram validar os critérios de adaptação do 
sequenciamento dos conteúdos. Entende-se que desta forma o STI desenvolvido potencializa o AVA Moodle, e pode se tornar um elemento auxiliar no processo ensinoaprendizagem.

\section{Referências}

ACAMPONA G.; GAETA M.; LOIA V. "Exploring e-Learning Knowledge Through Ontological Memetic Agents”. IEEE Computacional Intelligence Magazine. 2010.

AGUILAR, R.; MUÑHOZ V.; GONZÁLEZ E.J.; NODA M.; BRUNO A.; MORENO L. "Fuzzy and multiagent instructional planner for an intelligent tutorial system". Expert Systems with Applications, Elsevier. Science Direct. 2011.

AL-AJLAN A.; ZEDANS H. "Why Moodle". In International Workshop on Future Trends of Distributed Computing System. IEEE 2008.

BADARACCO, M.; MARTÍNEZ, L. "A fuzzy linguistic algorithm for adaptive test in intelligent tutoring system based on competences", Expert Systems with Applications, Elsevier. Science Direct. 2013.

BARRON, F. H. "Selecting a Best multi-attribute alternative with partial information about attribute weights". Acta Psychologica, n. 80, p. 91-103, 1992.

CHRYSAFIADI, K., VIRVOU, M. "Evaluating the integration of fuzzy logic into the student model of a web-based learning environment". Expert Systems with Applications, Elsevier. Science Direct. 2012.

NETTO, C.; GIRAFFA L. M. M.; FARIA E. T. "Graduações a Distância e o Desafio da Qualidade". ediPUCRS, Porto Alegre, 2010. Disponível em: $<$ http://www.pucrs.br/edipucrs/graduacoes.pdf $>$. Acesso em: 15/06/2014.

NOH, N. M.; AHMAD A; HALIM S A; ALI A. M. "Intelligent Tutoring System Using Rule-Based And Case-Based: A Comparison", In: The Third International Conference on e-Learning, ICEL2011, 23-24 November 2011, Bandung. 2012.

NUNES, T.; MUELlER, A.; TEIVE, R. C. G. "Ambientes Inteligentes de Aprendizagem: uma proposta baseada em Hipermídia Adaptativa e Redes Bayesianas". Anais do ICBL 2008 - Interactive Computer Aided Blended Learning. Florianópolis. 2008.

SUEBNUKARN, S.; HADDAWY, P. "COMET: A collaborative tutoring system for medical problem-based learning”. Intelligent Educational Systems, p. 1543-1673. IEEE. 2007.

WILGES B.; MATEUS G.P.; NASSAR S.M.; BASTOS R.C. "Integration of BDI agent with Fuzzy Logic in a Virtual Learning Environment". IEEE Latin America Transactions, Vol 10, No 1. 2012. 